Images dans le monde ibérique et ibéricoaméricain

5 | 2012

Le visible et l'invisible dans le monde hispanique et hispano-américain

\title{
«Pruebas de lo invisible » : sur quelques photographies de Manuel Álvarez Bravo
}

\section{Paul-Henri Giraud}

\section{(2) OpenEdition \\ Journals}

\section{Édition électronique}

URL : http://journals.openedition.org/agedor/891

DOI : 10.4000/agedor.891

ISSN : 2104-3353

Éditeur

Laboratoire LISAA

Référence électronique

Paul-Henri Giraud, " «Pruebas de lo invisible »: sur quelques photographies de Manuel Álvarez Bravo », L'Âge d'or [En ligne], 5 | 2012, mis en ligne le 01 mars 2012, consulté le 16 décembre 2019. URL : http:// journals.openedition.org/agedor/891 ; DOI : 10.4000/agedor.891 


\section{«PRUEBAS DE LO INVISIBLE» : SUR QUELQUES PHOTOGRAPHIES DE MANUEL ÁLVAREZ BRAVO}

Résumé : «Si tu veux voir l'invisible, observe soigneusement le visible», dit le Talmud. Le photographe mexicain Manuel Álvarez Bravo (1902-2002) aimait à citer cette phrase, et il ajoutait : « l'invisible est toujours contenu dans l'œuvre d'art, qui le recrée. S'il ne peut être vu en elle, l'œuvre d'art n'existe pas ». On pourrait illustrer ces affirmations avec beaucoup de photographies de don Manuel. Le présent article étudie plus particulièrement celles où les notions de vision, de viseur et de visite se trouvent explicitement signifiées par le titre. C'est le cas de quelques images classiques (Muchacha viendo pájaros, 1931 ; Caja de visiones, 1938) ou moins connues (La visita, 1935 ; Panteón, visitación, 1934-1965; No ves, 1994).

Mots-clés : photographie - Mexique - vision - mystique - humour - silence - Manuel Álvarez Bravo

Resumen: "Si quieres ver lo invisible, observa lo visible con cuidado", dice el Talmud. Al fotógrafo mexicano Manuel Álvarez Bravo (1902-2002) le gustaba citar esta frase, añadiendo: "lo invisible siempre está contenido en la obra de arte, que lo recrea. Si no puede ser visto en ella, la obra de arte no existe." Se podrían ilustrar estas afirmaciones con muchas fotografías de don Manuel. En este artículo, se estudian más particularmente algunas imágenes donde las nociones de visión, visor y visita se encuentran explicitadas por el título. Este es el caso de algunas fotos clásicas (Muchacha viendo pájaros, 1931; Caja de visiones, 1938) o menos conocidas (La visita, 1935; Panteón, visitación, 1934-1965; No ves, 1994).

Palabras clave : fotografía - México - visión - mística - humor - silencio - Manuel Álvarez Bravo 


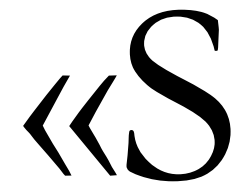

i tu veux voir l'invisible, observe soigneusement le visible », dit le Talmud. Le photographe mexicain Manuel Álvarez Bravo (1902-2002) aimait à citer cette phrase ${ }^{1}$. Pour le poète et critique Xavier Villaurrutia, son contemporain, c'est «cet accès à l'invisible par la route du visible » ${ }^{2}$ qui fait de certaines des images de don Manuel, à certains moments privilégiés, « de véritables preuves de l'invisible $»^{3}$.

La saveur mystique de ces affirmations est certainement paradoxale, s'agissant d'un art, la photographie, réputé s'en tenir strictement au visible. Mais la modernité esthétique n'aspire-t-elle pas à prendre le relais de la religion dans leur commune tentative d'exprimer l'inexprimable? Joyce - l'un des «dieux » ${ }^{4}$ d'Álvarez Bravo - enregistrait des «épiphanies »; Octavio Paz - l'un de ses amis -, scrutait la « révélation poétique ${ }^{5}$. Don Manuel recherche, sinon toujours la vision, du moins la suggestion d'une vision possible par delà le visible.

C'est ce que je vais tenter de montrer en examinant une série d'images dont certaines renvoient à l'univers visuel et conceptuel du catholicisme, dans sa variante mexicaine. Non que don Manuel soit un photographe chrétien (si tant est qu'une telle notion ait du sens); mais, ouvert à de multiples arts et littératures, et probablement assez éloigné de la religion de son enfance, il était sensible aux diverses manifestations de la piété populaire dans lesquelles il trouvait, non sans humour, des échos ou des métaphores de ses préoccupations esthétiques et philosophiques.

Je m’attacherai à des images des années 1920-1930, la période la plus connue de son œuvre, mais aussi à d'autres images, souvent méconnues, des années 1970-1990. Et j'aborderai le sujet difficilement appréhensible du visible et de l'invisible à travers trois termes voisins qui caractérisent le travail de ce photographe : vision - viseur - visite.

\footnotetext{
${ }^{1}$ Voir PEREZ, Nissan N., Dreams-Visions-Metaphors. The Photographs of Manuel Álvarez Bravo, Jérusalem, The Israel Museum, 1983, p. 19.

2 SHERIDAN, Guillermo, «Ojos en los ojos. Manuel Álvarez Bravo », in Manuel Álvarez Bravo. Ojos en los ojos / The Eyes in His Eyes, Santa Monica, Californie, Rosegallery / D.A.P., 2007, n. p.

3 VILLAURRUTIA, Xavier, «Manuel Álvarez Bravo» [1945], in Manuel Álvarez Bravo. Cien años, cien días, México, CONACULTA / INBA / Fundación Televisa / Fomento Cultural Banamex / Turner, 2001, p. 227.

${ }^{4}$ Sur cette ironique divinisation de l'artiste contemporain à travers le regard photographique d'Álvarez Bravo, voir SEGRE, Erica, "El retorno de Nuestros dioses (de Saturnino Herrán) en el modernismo fotográfico en México. Sincretismo estético, identidades amortajadas y reflexividad en un libro inédito de Manuel Álvarez Bravo ", article à paraittre in GIRAUD, Paul-Henri, RAMOS-IZQUIERDO, Eduardo, et RODRÍGUEZ, Miguel, 1910: México entre dos épocas, Mexico, El Colegio de México, 2013.

${ }^{5}$ Comme le dit le sous-titre du célèbre essai d'Octavio Paz : El arco y la lira. El poema, la revelación poética, poesía e historia (1956). - En 1982, Paz a consacré à Álvarez Bravo un texte critique (« Instante y revelación: Manuel Álvarez Bravo » in PAZ, Octavio, Los privilegios de la vista II. Arte de México, Obras completas, éd. de l'auteur, Barcelone, Círculo de Lectores, vol. 7, 1993, p. 312-317) et un poème ("Cara al tiempo. A Manuel Álvarez Bravo », in PAZ, Octavio, Obra poética II, Obras completas, op. cit., vol. 12, 2003, p. 53).
} 


\section{Vision}

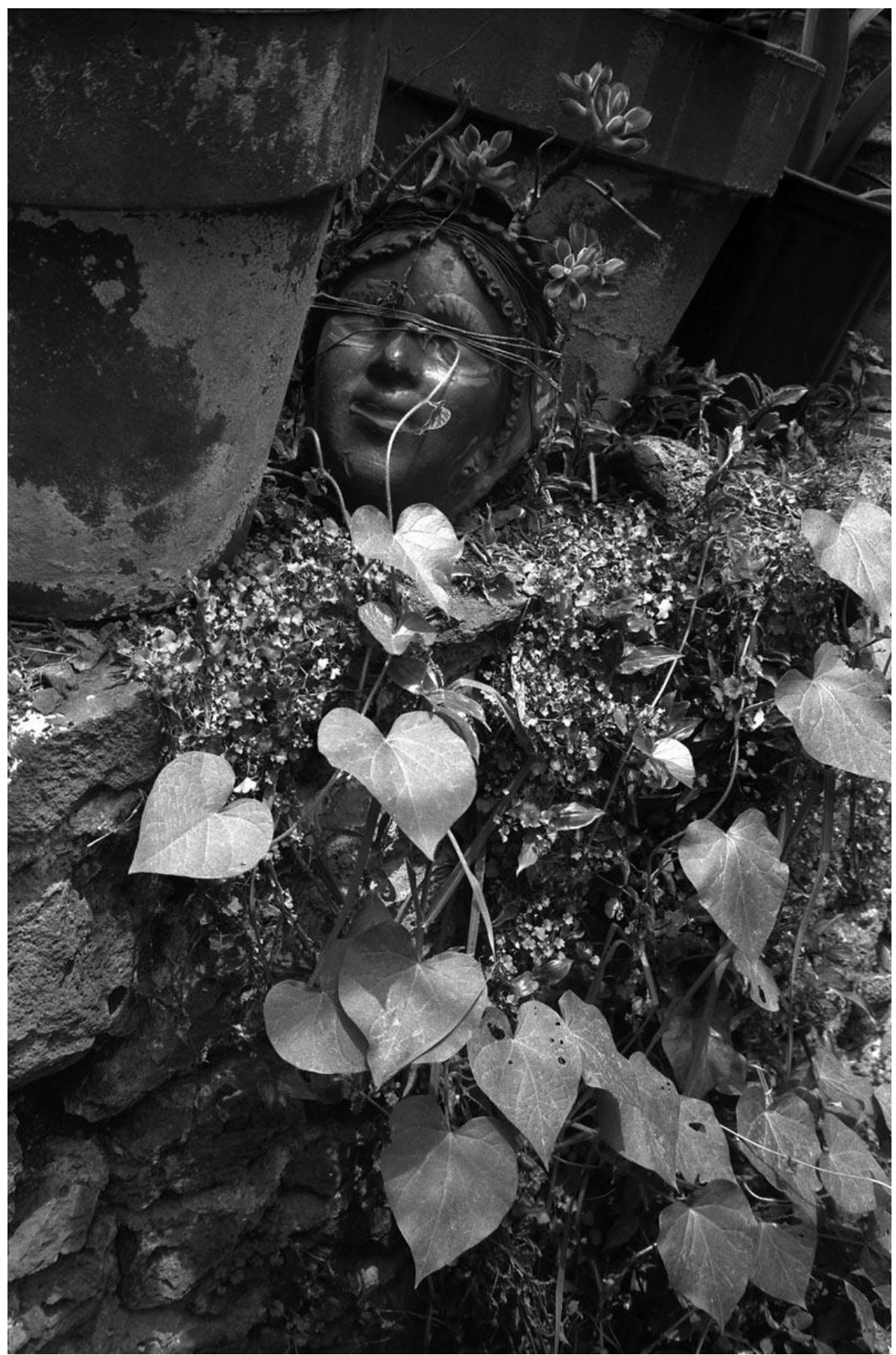

No ves, 1994 (C) Colette Urbajtel / Archivo Manuel Álvarez Bravo, S.C.

Une photographie prise en 1994, c'est-à-dire quand don Manuel avait quatre-vingt douze ans, s'intitule No ves: "Tu ne vois pas ». Cette phrase à la deuxième personne du singulier semble pouvoir s'appliquer à différents interlocuteurs. D'abord à la femme représentée par une sculpture, plus exactement une poterie, ici photographiée ; une poterie qui sert de récipient à une plante grasse. Ses yeux étant fermés, comme recouverts d'une bande translucide, et, de surcroit, en partie occultés par une ficelle, cette femme, en effet, ne 
voit pas. Deuxième destinataire possible de la phrase : l'artiste, le photographe lui-même qui, après une longue vie passée à regarder, à observer, à viser et à prendre des photos, serait encore comme un novice face au visible, se surprenant d'apercevoir ce qu'il n'avait pas vu, comme se reprochant à lui-même de ne pas voir. Enfin, la phrase "no ves » peut s'adresser au regardeur, à nous-mêmes, à qui l'artiste propose des images pour qu'enfin nous voyions. "Ils ont des yeux et ne voient pas ", écrivait saint Paul'. D'où l'intérêt de fermer les yeux pour voir, ou pour entendre, comme nous y invite Roland Barthes en ouvrant une parenthèse : «(fermer les yeux, c'est faire parler l'image dans le silence) $»^{7}$.

La formule «no ves» pourrait tout aussi bien s'appliquer à d'autres photographies d'Álvarez Bravo, ou à d'autres images qu'il affectionnait et qui faisaient partie de son musée intime, familier. Quand on pénètre dans la salle principale de sa maison, à Coyoacán, au sud de la mégalopole mexicaine - maison destinée à devenir un jour un musée -, lorsqu'on descend dans cette salle qui est une sorte de crypte de lave bien que de vastes baies vitrées y fassent pénétrer en abondance la lumière, lorsque l'on s'assoit dans cette pièce, on se retrouve face à face avec James Joyce, photographié à Paris en 1928 par Berenice Abbott ${ }^{8}$. Le chapeau de feutre porté par Joyce avait pour fonction, dit-on, d'atténuer la lumière qui atteignait ses yeux malades, menacés de perdre la vue. Mais comment ne pas voir que cette déficience oculaire est, chez un tel homme, proportionnelle à l'amplitude, à la richesse et à l'originalité de sa vision du monde et de la littérature ? La rêverie qu'exprime le regard intériorisé de James Joyce nous renvoie à la capacité de l'artiste de voir ou d'avoir des visions, des pensées, des pensées invisibles conçues au contact du visible. "Thought through my eyes...»- «Pensé à travers mes yeux...»: ainsi commence un chapitre d'Ulysse ("Protée »), et cette phrase est citée en exergue à un catalogue de photographies de Manuel Álvarez Bravo'. Face à ce portrait, on se dit que les yeux de Joyce lui servaient moins à voir qu'à penser.

Comme pour s'en souvenir, Álvarez Bravo fera lui-même le portrait de «James Joyce dans la bibliothèque » (vers 1980) ${ }^{10}$, où la photographie d'Abbott et une luxueuse édition en anglais de Ulysses voisinent avec des livres d'art présentant l'œuvre d'autres grands visionnaires : les Impressionnistes, Michel-Ange, le Greco ou Goya. "Hay tiempo » pouvait-on lire sur un bout de papier affiché dans le laboratoire du photographe. On peut comprendre : «il y a le temps », « on a le temps » ${ }^{11}$ - rien ne presse; « il y a du temps »- et la photographie est là pour en témoigner ; enfin, il y a le temps passé, toute une histoire de l'art qui s'accumule ici dans la bibliothèque et qui, comme Joyce ou encore Proust et quelques autres écrivains-opticiens - «Regardez vous-même si vous voyez mieux avec ce verre-ci, avec celui-là, avec cet autre $»^{12}-$ nous aide à mieux voir.

\footnotetext{
${ }^{6} \mathrm{Rm} 11,8$.

7 BARTHES, Roland, La Chambre claire. Note sur la photographie [1980], in CEuvres complètes, t. V, Livres, textes, entretiens. 1977-1980, Paris, Seuil, 2002, p. 833.

8 Photographie reproduite in GIRAUD, Paul-Henri, Manuel Álvarez Bravo. L'impalpable et l'imaginaire, Paris, Éditions de La Martinière, 2012, p. 115.

${ }^{9}$ Voir ÁLVAREZ BRAVO, Manuel, Fotografías 1928-1968, México, Comité Organizador de los XIX Juegos Olímpicos, 1968, p. 5.

${ }^{10}$ Photographie reproduite in GIRAUD, Paul-Henri, Manuel Álvarez Bravo, op. cit., p. 13.

11 «Quand il préparait l'exposition de Pasadena en 1971, Manuel Álvarez Bravo s'est retrouvé sans assistant pour tirer les épreuves. Le temps pressait et semblait insuffisant pour produire les tirages dans le laboratoire. Il écrivit alors quatre mots sur une feuillet qu'il fixa avec des punaises sur le mur de son laboratoire et qui l'accompagna jusqu'à sa mort en 2002: Hay tiempo ("On a le temps") / Hay tiempo ("On a le temps") ». GONZÁLEZ FLORES, Laura, in Manuel Alvarez Bravo, catalogue d'exposition, Madrid / Paris, Fundación Mapfre / Jeu de Paume / Hazan / Tf editores, 2012, p. 32.

12 PROUST, Marcel, Le Temps retrouvé, Paris, Gallimard, « Folio », 1990, p. 217-218.
} 
Manuel Álvarez Bravo devait particulièrement aimer Zurbarán. Dans une photo de $1986^{13}$, sainte Lucie, patronne des ophtalmologues, apparaît dénudée, mais également décapitée par le cadrage, les deux yeux dans son plat selon la représentation canonique ${ }^{14}$, et aussi, de façon plus étonnante, les deux seins détourés sur la peau par la trace du bain de soleil, comme en préparation d'un autre supplice, celui subi par une autre vierge sicilienne, sainte Agathe, que Zurbarán peignit portant dans un plateau ses «doux seins », "les plus dangereux ornements de l'incarnation », ainsi que l'écrivit le jeune Paul Valéry ${ }^{15}$. La LucieAgathe d'Álvarez Bravo porte donc à son comble le paradoxe de James Joyce : elle ne voit pas, elle ne voit plus le monde réel mais, grâce à son supplice, jouit certainement de la vision béatifique. "L'invisible», disait Álvarez Bravo, " est toujours contenu dans l'œuvre d'art, qui le recrée. S'il ne peut être vu en elle, l'œuvre d'art n'existe pas ${ }^{16}$. Moins qu'à l'artiste, dont en a vu qu'il pouvait être non pas «voyant» mais malvoyant, c'est à l'œuvre elle-même qu'il appartient de recréer l'invisible; c'est à la photographie, une fois développée (revelada, en espagnol), de faire surgir à nos yeux l'invisible pour que nous en soyons, peut-être, transformés.

La soif de voir unit l'artiste et le contemplateur. Le photographe ou le peintre peut choisir d'étancher cette soif en nous présentant des visages qui sont autant d'énigmes, de symboles de l'intériorité, d'« images de l'invisible », c'est-à-dire d'icônes, au sens que donnait à ce mot saint Jean Damascène, pourfendeur de l'iconoclasme et grand théoricien du culte des images ${ }^{17}$. Il peut aussi entretenir en nous cette soif du visage en le laissant hors champ. Le fragment de buste de Lucy offre ainsi le symbole d'une identité invisible qui fait appel à notre imagination, à notre réflexion, à notre extra-lucidité.

Dans la plupart des portraits, le modèle nous regarde, nous voit, et c'est dans l'eau de son regard que nous entrevoyons des abîmes. Mais le photographe peut aussi nous faire voir le regard que le modèle porte sur autre chose, et notamment sur un hors-champ; quitte à nous dire, dans le titre de la photo, ce que le modèle regarde, comme dans Muchacha viendo pájaros (1931) - image choisie par André Breton pour figurer sur la couverture du catalogue de l'exposition «Mexique » qu'il organisa à Paris en 1939 à la Galerie Renou et Colle ${ }^{18}$. L'intensité de ce regard et l'effroi pré-hitchkockien qu'il semble exprimer se trouvent rehaussés par l'éclat des clous qui ornent la porte de quelque église ou de quelque palais, porte contre laquelle la petite fille est assise. Sa robe en partie déchirée connote le danger qu'elle entrevoit dans ces oiseaux absents de l'image et d'autant plus présents dans notre imaginaire.

\footnotetext{
${ }^{13}$ Lucy, photographie reproduite sur le site www.manuelalvarezbravo.org/espagnol/ochenta.php.

${ }^{14}$ Cf. ZURBARÁN, Francisco de, Sainte Lucie, 1635-1640, huile sur toile, Chartres, Musée des Beaux-Arts, $115 \times 68 \mathrm{~cm}$.

15 VALÉRY, Paul, « Glose sur quelques peintures. Musée de Montpellier (1891) », in CEuvres complètes, Paris, Gallimard, «Bibliothèque de la Pléiade », t. II, 1960, p. 1289. Cf. ZURBARÁN, Francisco de, Sainte Agathe, vers 1634, huile sur toile, Montpellier, Musée Fabre, 129 x $61 \mathrm{~cm}$, et mon article « Entre pintura y fotografía. Redes intericónicas y juegos visuales en algunos desnudos de Manuel Álvarez Bravo ", in Rumbos del hispanismo en el umbral del Cincuentenario de la AIH, Actas del XVII Congreso de la Asociación Internacional de Hispanistas (Rome, juillet 2010), vol. VI, Stefano Tedeschi et Sergio Botta éd., Hispanoamérica, Rome, Bagatto Libri, 2012, p. 571577.

${ }^{16}$ Cité in PEREZ, Nissan N., Dreams-Visions-Metaphors, op. cit., p. 19.

17 Voir SENDLER, Egon, L'icône, image de l'invisible. Éléments de théologie, esthétique et technique, Paris, Desclée De Brouwer, coll. «Christus » $\mathrm{n}^{\circ}$ 54, 1981.

18 Photographie reproduite sur le site www.manuelalvarezbravo.org/espagnol/treinta-A.php. Cf. la couverture du catalogue de l'exposition de 1939 in GIRAUD, Paul-Henri, Manuel Álvarez Bravo, op. cit., p. 178.
} 
D'autres fois, le hors-champ n'est pas spécifié, simplement suggéré, comme dans Señor de Papantla (1934-1935) ${ }^{19}$. Et c'est alors le regard lui-même qui fait tout l'objet de la photo, le regard comme expression de la liberté et de la fierté de ce paysan de la région de Veracruz qui aurait évidemment toutes les caractéristiques souhaitées pour être la proie d'un photographe en quête de folklore, mais qui en l'occurrence semble se refuser avec obstination à regarder le photographe, et c'est ce refus même qui est photographié ${ }^{20}$. Le visible - c'est-à-dire, ici, toutes les données concrètes qui situent cette homme socialement, ethniquement, géographiquement, et en même temps hors du temps, enveloppé qu'il est par la lumière que filtrent les branches d'un arbre - est au service d'un invisible qui nous intrigue : que regarde cet homme? A-t-il vu le photographe ? Pourquoi ne nous regarde-t-il pas ? Se sent-il concerné ? Se sent-il visé?

19 Photographie reproduite in GIRAUD, Paul-Henri, Manuel Álvarez Bravo, op. cit., p. 131 et sur le site www.manuelalvarezbravo.org/espagnol/treinta-B.php.

${ }^{20}$ Hypothèse développée par John Mraz dans une conférence donnée le 28 novembre 2008 au siège de la Dirección de Estudios Históricos del INAH (Instituto Nacional de Arqueología e Historia), à Tlalpan, Mexico D. F., et reprise in MRAZ, John, Looking for Mexico. Modern Visual Culture and National Identity, Durham / Londres, Duke University Press, 2009, p. 87. 


\section{Viseur}

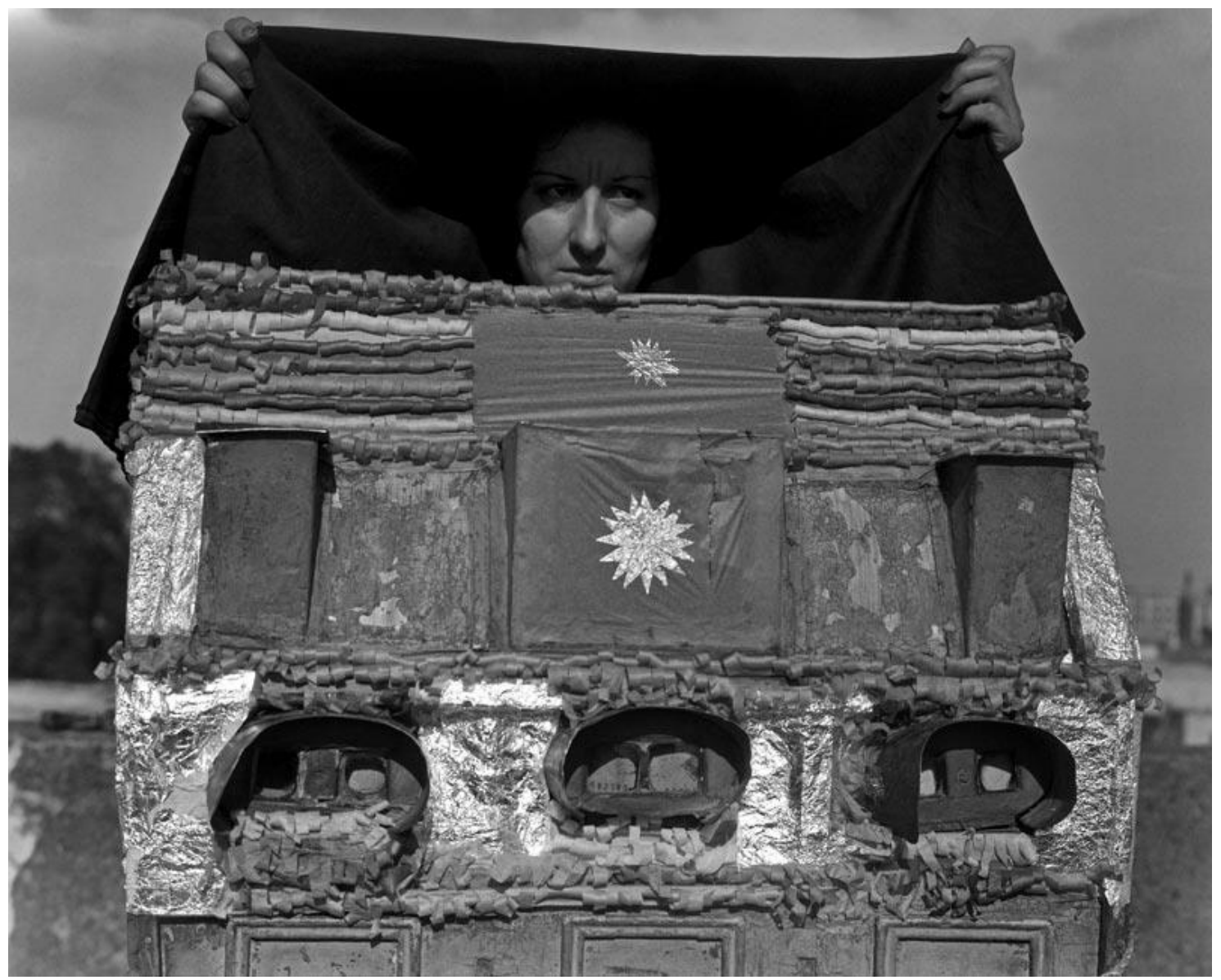

Caja de visiones, 1938 C Colette Urbajtel / Archivo Manuel Álvarez Bravo, S.C.

Pour visualiser l'invisible, le photographe dispose d'un auxiliaire : son appareil photo, et plus particulièrement son viseur.

Grâce au viseur, l'appareil peut en effet devenir une véritable «boîte à visions », un peu à la manière de l'artéfact rustique qui apparaît dans une image des années 30, Caja de visiones, véritable allégorie de la photographie. Cette image nous présente une de ces boîtes stéréoscopiques où l'on pouvait observer, depuis le $\mathrm{XIX}^{\mathrm{e}}$ siècle, des vues panoramiques. Mais il y a aussi, au-dessus de la boîte, le visage de Lola Álvarez Bravo, première épouse de don Manuel et elle-même photographe. Pareille apparition nous incite à voir dans cette boîte étrange une chambre photographique à l'ancienne, au moment où la tête de l'opératrice est sur le point d'être recouverte par le drap noir. Enfin, la superposition du visage et du drap dans le plan de l'image pourrait fournir la métaphore de l'épreuve photographique elle-même, comme le suggère Erica Segre ${ }^{21}$ en évoquant de prestigieux antécédents : le voile de sainte Véronique (patronne de la photographie), voile où le visage du Christ se serait imprimé, ou encore le manteau de Juan Diego, où la Vierge de Guadalupe a laissé son empreinte presque trois siècles avant l'invention de Nicéphore Niepce. L'appareil, l'acte et le résultat se trouvent donc réunis dans cette image à la fois

21 Voir SEGRE, Erica, Intersected Identities: Strategies of Visualization in Nineteenth-and Twentieth-Century Mexican Culture, New York and Oxford, Berghahn Books, coll. « Remapping Cultural History », 2007, p. 119. 
cocasse et mystique, nouvelle version de la Vera Icon, pastiche familier, familial, de la théologie chrétienne. Car si Lola («Dolores ») est l'avatar de la Vierge-Mère, précisons que son fils, son unique, se prénomme comme son père, Manuel («Dieu avec nous », nom révélé par l'ange de l'Annonciation), et que don Manuel, à l'instar du Père éternel des églises néo-classiques, se représentait volontiers sous la forme d'un œil...

C’est souvent l'œil près du viseur que Manuel Álvarez Bravo se laisse entrevoir dans les autoportraits dont il n'était pas coutumier, au départ, mais qu'il multiplie à la fin de sa vie, comme pour laisser une trace visible de lui-même au moment où il va devenir invisible en passant de l'autre côté du miroir. Dans son autoportrait dans le rétroviseur ${ }^{22}$ (1996), il juxtapose les deux yeux: l'un, naturel, en haut, à droite; l'autre, mécanique, au-dessous. Dans tel autre autoportrait ${ }^{23}$ (1995), son image se fragmente dans plusieurs miroirs comme pour rappeler la multiplicité insaisissable du visible ; le voici dans la cour de son atelier, à proximité de plusieurs signes de l'invisible : une idole préhispanique, à gauche ; une échelle, à droite - symbole simple d'une ascension vers la vision, vers l'illumination, peut-être, bien que l'ampoule tout en haut du mur soit éteinte.

Ce que montre la photographie d'Álvarez Bravo, c'est donc moins la vision ellemême que le chemin qu'il faut faire pour y accéder. Les barreaux d'une échelle qui mène en haut d'un mur ${ }^{24}$. L'œil-de-bœuf d'une lucarne par laquelle "la fille des danseurs ${ }^{25}$, dressée sur la pointe des pieds, appuyée, elle aussi, contre un mur, auquel un motif à chevrons clairs et foncés imprime un rythme ascensionnel, épie quelque spectacle qui nous est à jamais dérobé. Per visibilia ad invisibilia ${ }^{26}$ : tel est le chemin ordinaire de la mystique photographique.

Or, sur ce chemin, la visite de certains lieux ou à des personnes précises est une étape utile et peut-être nécessaire.

\footnotetext{
22 Photographie reproduite sur le site www.manuelalvarezbravo.org/espagnol/noventa.php.

23 Photographie reproduite in GIRAUD, Paul-Henri, Manuel Álvarez Bravo, op. cit., p. 123 et sur le site www.manuelalvarezbravo.org/espagnol/noventa.php.

24 Voir Para subir al cielo (1970-1972), in www.manuelalvarezbravo.org/espagnol/setenta.php.

25 Photographie reproduite in GIRAUD, Paul-Henri, Manuel Alvarez Bravo, op. cit., p. 70 et sur le site www.manuelalvarezbravo.org/espagnol/treinta-A.php.

${ }^{26}$ Formule inspirée par saint Paul (2 Cor. 4,18) à saint Thomas d'Aquin (Somme théologique I, question 43,7).
} 


\section{Visite, visitation}

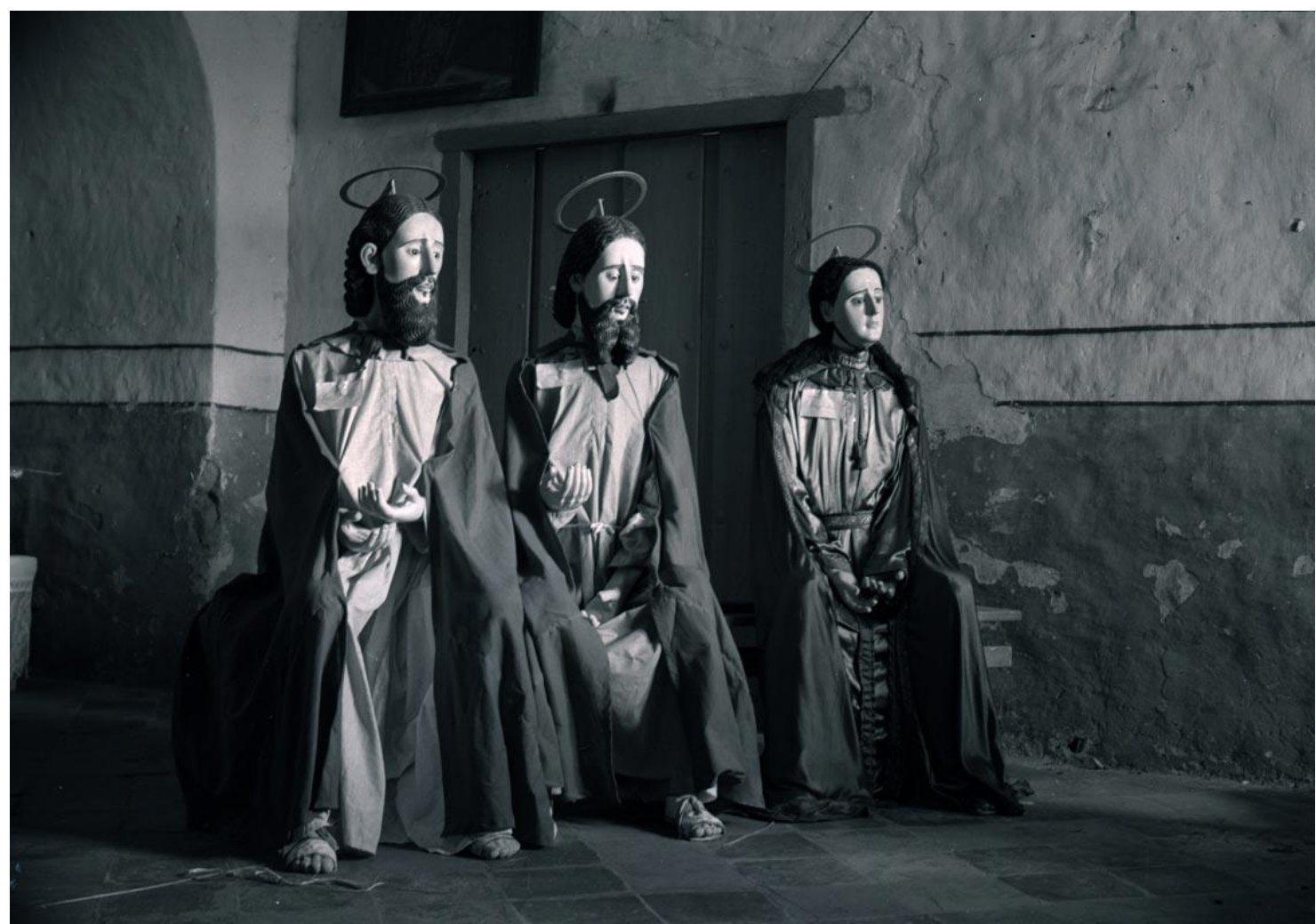

La visita, 1935 C Colette Urbajtel / Archivo Manuel Álvarez Bravo, S.C.

Manuel Álvarez Bravo aime depuis toujours les bultos, ces statues religieuses qu'on porte en procession ou qui servent à représenter des épisodes évangéliques. Vers 1925, il en photographiait, à Oaxaca ${ }^{27}$. En 1935, dans le Michoacán, il photographie trois statues assises dans un couvent ou une église, dans une salle ancienne et sobre aux murs en partie décrépis qui pourrait être, aussi bien, la salle à manger d'une vieille hacienda. La photo s'intitule La visita, et l'on a, en effet, la curieuse impression d'une visite profane où trois personnes se tiennent en silence, dans un de ces abîmes qui ponctuent une conversation languissante.

Lola Álvarez Bravo était du voyage puisque, sous un autre angle, elle a capté les mêmes personnages dans une mise en scène encore inachevée de La última cena - l'autre titre de la photo, Pocos los escogidos, semblant faire allusion au fait que les apôtres ne sont pas au complet ${ }^{28}$. En comparant cette image à La visita, mais aussi à une autre photo de don Manuel, Los Creadores, los Formadores ${ }^{29}$ (titre énigmatique s'il en est, tiré du Popol Vuh, et faisant référence aux dieux créateurs de la cosmologie maya), on comprend que le couple de photographes a assisté, ce jour-là, à la mise en place des statues autour d'une table pour

${ }^{27}$ Voir Corona de espinas, 1925. Photographie reproduite in GIRAUD, Paul-Henri, Manuel Álvarez Bravo, op. cit., p. 28 et sur le site www.manuelalvarezbravo.org/espagnol/veinte.php.

28 Photographie reproduite in FERRER, Elizabeth, Lola Alvarez Bravo, Mexico, Fondo de Cultura Económica / Turner, 2006, p. 30-31. Titres, lieu et date sont précisés p. 157 : «La última cena (Pocos los escogidos), Erongarícuaro, Michoacán, ca. $1935 »$.

${ }^{29}$ Bien que le site www.manuelalvarezbravo.org/espagnol/cuarenta.php date cette photo de 1940-1942, je fais l'hypothèse que, comme les deux images précédentes, elle est de 1935. 
une représentation de la Dernière cène, probablement en vue de la célébration du Jeudi saint. Or si la Cène est le moment de l'institution de l'Eucharistie, c'est-à-dire, pour le christianisme, de la manifestation par excellence de l'Invisible dans le monde visible, la photo La visita se situe volontairement en deçà de cette manifestation, dans un entre-deux temporel, dans l'espace incertain d'une pièce à demi vide, dans l'attente de quelque chose, mais on ne sait de quoi. Loin de diminuer le caractère métaphysique de ces statues, l'attente l'augmente en l'indéterminant. Les voici qui attendent d'être rejointes, d'être "visitées », animées peut-être, mais par qui ou par quoi ?

Le caractère elliptiquement religieux de l'art de Manuel Álvarez Bravo apparaît encore dans ses nombreuses photographies de tombes, lesquelles sont, au Mexique, des lieux très visités. Dans L'esprit des personnes ${ }^{30}$ (1927), la lueur fragile des cierges sur le point de s'éteindre est le signe visible de deux personnes invisibles : le mort, enseveli ; le visiteur, peut-être déjà parti. Le signe de la pénétration irréversible de l'esprit du défunt dans le règne de l'invisible, et peut-être aussi de l'oubli de ceux qui demeurent en vie. Mais une photographie de 1964-1965 nous donne une vision moins mélancolique de la mort. Son titre duel - Panteón, visitación ${ }^{31}$ - fait allusion, d'abord, à la visite qu'une très jeune fille fait à une tombe encore fraîche, ornée de fleurs à la manière de la campagne mexicaine. Si le mort, invisible, se laisse imaginer, un autre personnage, à peine visible, se laisse voir dans le coin gauche de la photo: un jeune homme derrière le mur. Les regards des deux jeunes gens ne se croisent pas mais l'on devine entre eux une relation implicite ; une histoire qui commence, peut-être. Le titre de la photo laisse entendre que cette commune visite au cimetière pourrait être l'occasion d'une rencontre, d'une intime reconnaissance, d'une invisible conjonction entre ces deux êtres : moment propice à une visitation.

Vision, viseur, visite, visitation, visage ${ }^{32}$ : la présence ou l'absence de ces éléments scande l'œuvre d'Álvarez Bravo depuis son début jusqu'à son terme, comme le montrent encore les polaroïds réalisés dans son grand âge, sur un mode plutôt ludique, et qui ont fait l'objet d'un livre posthume publié en 2005. Malgré les limites de ce procédé couleur - ou grâce à ces limites mêmes - s'éveillent des échos de l'œuvre en noir et blanc. On y retrouve le goût pour les statues polychromes des églises mexicaines, et cette manière de couper la tête des figures pour mieux attirer notre attention sur le corps - sur la peau sensible aux coups et aux blessures comme la pellicule l'est à la lumière -, pour nous faire mieux désirer, peutêtre, le visage absent du modèle ${ }^{33}$. On y trouve également, comme à l'accoutumée, des paysages énigmatiques ${ }^{34}$, mais ici, pour une fois, avec un abandon au lyrisme de la forme et de la couleur, dans telle image naïve et lumineuse où un nuage rouge s'allonge par-dessus le toit $^{35}$. On y découvre enfin - rareté - une photo floue, où un nu féminin, assis, sans pieds ni tête, nous fait désirer d'en voir plus et d'en savoir plus ${ }^{36}$.

\footnotetext{
30 Photographie reproduite in GIRAUD, Paul-Henri, Manuel Álvarez. Bravo, op. cit., p. 74 et sur le site www.manuelalvarezbravo.org/espagnol/veinte.php.

31 Photographie reproduite in GIRAUD, Paul-Henri, Manuel Álvarez Bravo, op. cit., p. 79 et sur le site www.manuelalvarezbravo.org/espagnol/sesenta.php.

32 Sur l'approche du visage par Álvarez Bravo et la polysémie du mot «retrato» dans son œuvre, voir GIRAUD, Paul-Henri, Manuel Álvarez. Bravo, op. cit., p. 108-141.

33 Photographie reproduite in ÁLVAREZ BRAVO, Manuel, Polaroids, Mexico, RM, 2005, p. 10.

${ }^{34}$ Cf. par exemple "Parvada sobre el mar», 1939 (in www.manuelalvarezbravo.org/espagnol/treinta-B.php), «Soledad primera », 1956, et "Lago de Texcoco 3 », 1962-1964, in GIRAUD, Paul-Henri, Manuel Álvarez. Bravo, op. cit., p. 216-218.

35 ÁLVAREZ BRAVO, Manuel, Polaroids, op. cit., p. 23.

36 Ibid., p. 93.
} 
«La musique savante manque à notre désir " ${ }^{37}$, écrivait Rimbaud. Et Roland Barthes : «La photographie doit être silencieuse [...] : ce n'est pas une question de "discrétion", mais de musique $»^{38}$. Manuel Álvarez Bravo, grand mélomane, est l'auteur d'une œuvre d'autant plus «savante » qu'elle aiguise notre désir d'une visualité plénière, à partir de ce qui, dans l'expérience quotidienne, n'est guère jamais qu'entrevu.

${ }^{37}$ Dernière phrase du poème «Conte » in Les illuminations.

${ }^{38}$ BARTHES, Roland, La Chambre claire, in CEuvres complètes, t. V, op. cit., p. 833. 